\title{
A Concise Proof of the Littlewood-Richardson Rule
}

\author{
John R. Stembridge* \\ Department of Mathematics \\ University of Michigan \\ Ann Arbor, Michigan 48109-1109 USA \\ jrs@umich.edu
}

Submitted January 2, 2002; Accepted March 15, 2002

MR Subject Classification: 05E05

\begin{abstract}
We give a short proof of the Littlewood-Richardson rule using a sign-reversing involution.
\end{abstract}

\section{Introduction.}

The Littlewood-Richardson rule is one of the most important results in the theory of symmetric functions. It provides an explicit combinatorial rule for expressing either a skew Schur function, or a product of two Schur functions, as a linear combination of (non skew) Schur functions. Since Schur functions in $n$ variables are the irreducible polynomial characters of $G L_{n}(\mathbf{C})$, the Littlewood-Richardson rule amounts to a tensor product rule for $G L_{n}(\mathbf{C})$.

The rule was first formulated in a 1934 paper by Littlewood and Richardson $[\mathbf{L R}]$, but the first complete proofs were not published until the 1970's. (For a historical account of the evolution of the rule and its proofs, see the recent survey paper of van Leeuwen $[\mathbf{v L}]$.) There are now many proofs available, such as those based on the Robinson-Schensted-Knuth correspondence, jeu de taquin, or the plactic monoid. In this note, we present a very simple, self-contained proof of the rule; the argument also proves at the same time the "bi-alternant" formula for Schur functions- the formula originally used by Cauchy to define Schur functions.

We obtained this proof by specializing a crystal graph argument that works in much greater generality (see Theorem 2.4 of $[\mathbf{S}]$ ). The fact that crystal graphs (or the closely related Path Model of Littelmann) may be used to prove the Littlewood-Richardson rule, as well as tensor product rules for other semisimple Lie groups, is well-known (see [KN] or $[\mathbf{L}]$ ), but we believe that it is not widely understood that there exist versions of these proofs that are self-contained, with no need to appeal to a general theory.

The proof we present here is not the first short proof. Alternatives include proofs by Berenstein and Zelevinsky $[\mathbf{B Z}]$, Remmel and Shimozono $[\mathbf{R S}]$, and Gasharov $[\mathbf{G}]$. Furthermore, aside from the differences in language between semistandard tableaux and Gelfand patterns, the sign-reversing involution we use here is essentially a translation of the one used by Berenstein and Zelevinsky.

* Work supported by NSF grant DMS-0070685. 


\section{The Details.}

Let $\mathcal{P}$ denote the set of nonnegative integer sequences of the form $\lambda=\left(\lambda_{1} \geq \lambda_{2} \geq \cdots\right)$ with finitely many nonzero terms; i.e., the set of partitions. We let $\mathcal{P}_{n}$ denote the set of partitions with at most $n$ nonzero terms, viewed (by truncation) as a subset of $\mathbf{Z}^{n}$.

Now regard $n$ as fixed, and set $\rho=(n-1, \ldots, 1,0)$ and $\varnothing=(0, \ldots, 0) \in \mathcal{P}_{n}$.

For each $\lambda \in \mathbf{Z}^{n}$, define $x^{\lambda}=x_{1}^{\lambda_{1}} \cdots x_{n}^{\lambda_{n}}$ and $a_{\lambda}=\operatorname{det}\left[x_{i}^{\lambda_{j}}\right]=\sum_{w \in S_{n}} \operatorname{sgn}(w) x^{w \lambda}$.

Given $\mu, \nu \in \mathcal{P}$, let $D(\mu, \nu)=\left\{(i, j) \in \mathbf{Z}^{2}: 1 \leq i \leq n, \nu_{i}<j \leq \mu_{i}\right\}$. Assuming $\nu \leq \mu$ (meaning $\nu_{i} \leq \mu_{i}$ for all $i$ ), define $\mathcal{S}(\mu / \nu)$ to be the set of semistandard tableaux of shape $\mu / \nu$; i.e., the set of mappings $T: D(\mu, \nu) \rightarrow[n]$ with increasing columns $(T(i, j)<T(i+1, j))$ and weakly increasing rows $(T(i, j) \leq T(i, j+1))$. The weight of $T$ is $\omega(T)=\left(\omega_{1}(T), \ldots, \omega_{n}(T)\right) \in \mathbf{Z}^{n}$, where $\omega_{k}(T)=\left|T^{-1}(k)\right|$ denotes the number of $k^{\prime}$ 's in $T$. The generating series $s_{\mu / \nu}=\sum_{T \in \mathcal{S}(\mu / \nu)} x^{\omega(T)}$ is a skew Schur function.

There is a well-known set of involutions $\sigma_{1}, \ldots, \sigma_{n-1}$ on $\mathcal{S}(\mu / \nu)$, due to Bender and Knuth $[\mathbf{B K}]$, with the property that $\sigma_{k}$ acts by changing certain entries of $T \in \mathcal{S}(\mu / \nu)$ from $k$ to $k+1$ and vice-versa in such a way that $\omega\left(\sigma_{k}(T)\right)=s_{k} \omega(T)$, where $s_{k}$ denotes the transposition $(k, k+1) \in S_{n}$. The existence of these involutions proves that $s_{\mu / \nu}$ is a symmetric function of $x_{1}, \ldots, x_{n}$.

To explicitly describe the action of $\sigma_{k}$ on $T \in \mathcal{S}(\mu / \nu)$, declare an entry $k$ or $k+1$ to be free in $T$ if there is no corresponding $k+1$ or $k$ (respectively) in the same column. It is easy to check that the free entries in a given row must occur in consecutive columns; moreover, the entries in the free positions may be arbitrarily changed from $k$ to $k+1$ and vice-versa without violating semistandardness as long as the free positions remain weakly increasing by row. The tableau $\sigma_{k}(T)$ is obtained by reversing the numbers of free $k$ 's and $k+1$ 's within each row; i.e., if there are $a_{i}$ free $k$ 's and $b_{i}$ free $k+1$ 's in row $i$ of $T$, then there should be $b_{i}$ free $k$ 's and $a_{i}$ free $k+1$ 's in row $i$ of $\sigma_{k}(T)$.

In the following, $T_{\geq j}$ denotes the subtableau of $T$ formed by the entries in columns $j, j+1, \ldots$, and we use similar notations such as $T_{<j}$ and $T_{>j}$ in the obvious way.

Theorem. For all $\lambda \in \mathcal{P}_{n}$ and all $\mu, \nu \in \mathcal{P}$ such that $\nu \leq \mu$, we have

$$
a_{\lambda+\rho} s_{\mu / \nu}=\sum a_{\lambda+\omega(T)+\rho}
$$

where the sum ranges over all $T \in \mathcal{S}(\mu / \nu)$ such that $\lambda+\omega\left(T_{\geq j}\right) \in \mathcal{P}_{n}$ for all $j \geq 1$.

Proof. As noted above, we know that $s_{\mu / \nu}$ is symmetric, so for each $w \in S_{n}$, the quantities $w(\lambda+\rho)+\omega(T)$ and $w(\lambda+\rho+\omega(T))$ are identically distributed as $T$ varies over $\mathcal{S}(\mu / \nu)$. Hence,

$$
a_{\lambda+\rho} s_{\mu / \nu}=\sum_{w \in S_{n}} \sum_{T \in \mathcal{S}(\mu / \nu)} \operatorname{sgn}(w) x^{w(\lambda+\rho+\omega(T))}=\sum_{T \in \mathcal{S}(\mu / \nu)} a_{\lambda+\omega(T)+\rho} .
$$

We declare $T$ to be a Bad Guy if $\lambda+\omega\left(T_{\geq j}\right)$ fails to be a partition for some $j$; i.e.,

$$
\lambda_{k}+\omega_{k}\left(T_{\geq j}\right)<\lambda_{k+1}+\omega_{k+1}\left(T_{\geq j}\right)
$$


for some pair $k, j$. Among all such pairs $k, j$, choose one that maximizes $j$, and among those, choose the smallest $k$. It must be the case that $\lambda+\omega\left(T_{>j}\right)$ is a partition, and since $\omega_{k}\left(T_{\geq j}\right)-\omega_{k+1}\left(T_{\geq j}\right)$ can change by at most one if we increment or decrement $j$, there must be a $k+1$ in column $j$ of $T$ (and no $k$ ), and

$$
\lambda_{k}+\omega_{k}\left(T_{\geq j}\right)+1=\lambda_{k+1}+\omega_{k+1}\left(T_{\geq j}\right) .
$$

Let $T^{*}$ denote the tableau obtained from $T$ by applying the Bender-Knuth involution $\sigma_{k}$ to the subtableau $T_{<j}$, leaving the remainder of $T$ unchanged. Since this involves changing some subset of the entries of $T_{<j}$ from $k$ to $k+1$ and vice-versa, and column $j$ has a $k+1$ but no $k$, it is easy to see that $T^{*}$ is semistandard. Furthermore, $\left(T^{*}\right)_{\geq j}$ and $T_{\geq j}$ are identical, so $T \mapsto T^{*}$ is an involution on the set of Bad Guys. In comparing the contributions of $T$ and $T^{*}$ to (1), note that $s_{k} \omega\left(T_{<j}\right)=\omega\left(T_{<j}^{*}\right)$, whereas (2) implies that $s_{k}$ fixes $\lambda+\omega\left(T_{\geq j}\right)+\rho$, whence $s_{k}(\lambda+\omega(T)+\rho)=\lambda+\omega\left(T^{*}\right)+\rho$ and

$$
a_{\lambda+\omega(T)+\rho}=-a_{\lambda+\omega\left(T^{*}\right)+\rho} .
$$

The contributions of Bad Guys may therefore be canceled from (1).

For the shape $\mu=\mu / \varnothing$, we have $\omega\left(T_{\geq j}\right) \in \mathcal{P}_{n}$ for all $j$ only if every entry in row $i$ of $T$ is $i$; thus, there is a unique such $T$, it has weight $\mu$, and hence $a_{\rho} s_{\mu}=a_{\mu+\rho}$, or

Corollary (The Bi-Alternant Formula). For all $\mu \in \mathcal{P}_{n}$, we have $s_{\mu}=a_{\mu+\rho} / a_{\rho}$.

Corollary. For all $\lambda \in \mathcal{P}_{n}$ and all $\mu, \nu \in \mathcal{P}$ such that $\nu \leq \mu$, we have

$$
s_{\lambda} s_{\mu / \nu}=\sum s_{\lambda+\omega(T)},
$$

where the sum ranges over all $T \in \mathcal{S}(\mu / \nu)$ such that $\lambda+\omega\left(T_{\geq j}\right) \in \mathcal{P}_{n}$ for all $j \geq 1$.

This corollary is Zelevinsky's extension of the Littlewood-Richardson rule $[\mathbf{Z}]$.

Taking the specialization $\lambda=\varnothing$, we obtain the decomposition of $s_{\mu / \nu}$ into Schur functions; it is simpler than the traditional formulation of the Littlewood-Richardson rule as found (e.g.) in $[\mathbf{M}]$, since it does not involve converting tableaux to words and imposing the "lattice permutation" condition. However, it still involves counting semistandard tableaux of shape $\mu / \nu$ satisfying certain properties, and it is a not-toodifficult exercise to show that these two formulations count the same tableaux.

Via the specialization $\nu=\varnothing$, we obtain yet another formulation of the LittlewoodRichardson rule - in this case involving the decomposition of $s_{\lambda} s_{\mu}$ into Schur functions. 


\section{References.}

[BK] E. A. Bender and D. E. Knuth, Enumeration of plane partitions, J. Combin. Theory Ser. A 13 (1972), 40-54.

[BZ] A. D. Berenstein and A. V. Zelevinsky, Involutions on Gelfand-Tsetlin schemes and

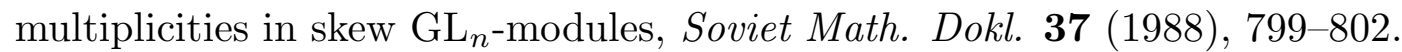

[G] V. Gasharov, A short proof of the Littlewood-Richardson rule, European J. Combin. 19 (1998), 451-453.

[KN] M. Kashiwara and T. Nakashima, Crystal graphs for representations of the $q$ analogue of classical Lie algebras, J. Algebra 165 (1994), 295-345.

[L] P. Littelmann, A Littlewood-Richardson rule for symmetrizable Kac-Moody algebras, Invent. Math. 116 (1994), 329-346.

[M] I. G. Macdonald, "Symmetric Functions and Hall Polynomials," Second Edition, Oxford Univ. Press, Oxford, 1995.

[LR] D. E. Littlewood and A. R. Richardson, Group characters and algebra, Phil. Trans. A 233 (1934), 99-141.

[RS] J. B. Remmel and M. Shimozono, A simple proof of the Littlewood-Richardson rule and applications, Discrete Math. 193 (1998), 257-266.

[S] J. R. Stembridge, Combinatorial models for Weyl characters, Advances in Math., to appear.

[vL] M. A. A. van Leeuwen, The Littlewood-Richardson rule, and related combinatorics, in "Interactions of Combinatorics and Representation Theory," MSJ Memoirs 11, Math. Soc. Japan, Tokyo, 2001, pp. 95-145.

[Z] A. V. Zelevinsky, A generalization of the Littlewood-Richardson rule and the Robinson-Schensted-Knuth correspondence, J. Algebra 69 (1981), 82-94. 